\title{
Three-body wear of luting composites and influence of the ACTA wheel material
}

\author{
Lea HOFFMANN, Andreas KESSLER and Karl-Heinz KUNZELMANN \\ Department of Conservative Dentistry and Periodontology, University Hospital, LMU Munich, Munich, Germany \\ Corresponding author, Lea HOFFMANN; E-mail: lea.hoffmann@med.uni-muenchen.de
}

\begin{abstract}
The aim of the study was to investigate the wear of luting materials (Variolink Esthetic LC/DC, Multilink Automix, Panavia V5 paste) compared to a resin composite (Tetric EvoFlow) with an ACTA-machine (three-body-wear simulator) using steel and 3Dprinted polymer wheels. Wear was measured (software Match3D) and statistically analyzed (ANOVA). Worn surface was examined with a field-emission-scanning-electron-microscope. Finite element analysis was carried out to analyze differences in the mechanical stress of the two different sample wheels. Using the steel wheel, Variolink Esthetic DC exhibited significantly less wear than Tetric EvoFlow after 200,000 cycles $(p=0.037)$. Luting composites on polymer wheels exhibited significantly lower wear values compared to the steel wheels $(p<0.001)$. Finite element analysis showed higher stresses for the steel wheel compared to the polymer wheel. Mechanical properties of the sample wheels had a high influence on wear properties. Dual- or auto-polymerizing materials seem to have a small advantage over the light-curing materials.
\end{abstract}

Keywords: Luting agents, Three-body-wear, 3D printing, ACTA, Finite element analysis

\section{INTRODUCTION}

Optimal marginal fit of dental restorations is crucial for their long-term success ${ }^{1,2)}$. Secondary caries and loss of stability are still the most frequent causes of failure of fixed restorations ${ }^{3)}$. The marginal gap between the tooth and indirect restorations, which is filled by the luting material, still represents a vulnerable area. Furthermore, wear of the luting material is a major contributing factor to complications ${ }^{4-77}$. Loss of marginal integrity may lead to accumulation of plaque, marginal staining, in case of exposed dentin even to postoperative hypersensitivity or secondary caries ${ }^{8,9}$. Thus, a small marginal gap of $<120 \mu \mathrm{m}$ and a proper selection of the luting material are considered to be important steps to ensure the long-term success of dental restorations ${ }^{10,11}$.

Wear resistance is one of the most important factors for the functionality of restorations in the oral cavity. In contrast to material properties such as elastic modulus, hardness or flexural strength, wear is a system property that results from the complex interaction of various factors of the tribosystem ${ }^{12)}$. Generally, there are two types of wear to distinguish: two-body wear and threebody wear ${ }^{13)}$. The two-body wear displays the masticatory activity as it consists of an agonist and antagonist sliding against each other ${ }^{14)}$. Three-body wear involves a freely movable third medium (abrasive particles) trapped between two solid surfaces ${ }^{13)}$. Three-body wear plays an even more important role in dental wear analysis, especially considering the marginal gap of fixed dental restorations. The time a restorative material comes into direct contact with an opposing tooth or restoration (two-body wear) is quite minimal, compared to contact with a food bolus or other third body (three-body wear) such as toothpaste ${ }^{15}$.

Due to the increased time required, the costs, the biological range of variation and the difficulty of quantifying wear in vivo, early attempts were made to develop experimental setups and in vitro machines to simulate oral conditions and to predict the wear behavior of various dental materials ${ }^{16-18)}$. In 1986, De Gee developed a three-body wear simulator (ACTA- Academic Center for Dentistry Amsterdam). By including test parameters such as contact pressure, slip, intermediate medium and speed of movement De Gee achieved comparable wear values to clinical investigations ${ }^{19,20)}$. In addition, a series of chewing simulators (two-body wear simulators) have been developed during the last 30 years. Fang has shown that using a two-body wear chewing simulator the amount of wear is significantly influenced by material, the shape and the surface treatment of the antagonist ${ }^{21}$. Enamel antagonists showed the most realistic wear simulation in previous investigations. However, large variations in tooth shape leads to considerable scatter of the results. Another frequently used synthetic antagonist material is steatite, as it resembles the hardness of enamel. However, relatively large steatite wear particles are produced in the wear simulation due to the microstructure of steatite, turning two-body wear into three-body wear ${ }^{22)}$.

Based on these findings we decided to use the ACTA wear machine for the present study as it remains a standard method for reliably investigating wear values of dental materials of variable composition and different mechanical properties ${ }^{12}$.

The initial study objective was to investigate the wear resistance of the most common luting composites with the ACTA wear simulation. Instead of the conventional steel wheel, the ACTA sample wheels were 3D-printed with an acrylate resin (Form2, Formlabs, Somerville, MA, USA). Polymer wheels are less expensive, offer greater flexibility in the number and shape of material 
chambers and, unlike steel wheels, can be ground down together with the test material. However, in our preliminary investigations, this modified set up using polymer wheels showed no meaningful outcomes in the first run. The modified set-up exhibited much lower wear values in contrast to wear values of comparable resins published in previous studies ${ }^{19,23)}$. The findings suggested that the material of the ACTA sample wheels could have an influence on the wear behavior of the investigated materials.

Therefore, the aim of the present study was i) to investigate the wear resistance of the most common luting materials and ii) to analyze the influence on the wear behavior of 3D-printed polymer wheels compared to the commonly used steel wheels.

\section{MATERIALS AND METHODS}

Table 1 summarizes the materials investigated and their compositions as they are commercially available. Materials included light-curing (Variolink Esthetic LC, Ivoclar Vivadent, Schaan, Liechtenstein) and dual-curing [Variolink Esthetic DC (Ivoclar Vivadent), Multilink Automix (Ivoclar Vivadent), Panavia V5 paste (Kuraray, Tokyo, Japan)] luting agents. A resin composite [Tetric EvoFlow (Ivoclar Vivadent)] was selected as reference material. The three body-wear was investigated in an ACTA-machine, which simulates wear caused by food using a millet seed suspension that is pressed onto the sample surface by two antagonistic wheels.

In contrast to the classical test setup, not only ACTA steel wheels were used but also 3D-printed polymer wheels (two commonly used steel wheels and two polymer wheels). For producing the 3D-printed wheels, a computer-assisted STL file was created according to the dimension of the steel wheel using the design software SolidWorks (Dassault Systèmes, Vélizy-Villacoublay, France). Afterward, the STL file was imported into

Table 1 Brand names, manufacturers, composition (according to manufacturer), and batch numbers of materials tested

\begin{tabular}{|c|c|c|c|c|c|c|}
\hline Material & Manufacturer & Matrix & Filler & wt $\% /$ vol\% & Filler-size & Reference \\
\hline $\begin{array}{l}\text { Variolink } \\
\text { Esthetic } \\
\text { LC }\end{array}$ & $\begin{array}{l}\text { Ivoclar } \\
\text { Vivadent, } \\
\text { Schaan, } \\
\text { Liechtenstein }\end{array}$ & $\begin{array}{l}\text { urethane dimethacrylate } \\
\text { (UDMA), methacrylate } \\
\text { monomers, inorganic fillers, } \\
\text { initiators and stabilizers, } \\
\text { pigments }\end{array}$ & $\begin{array}{l}\text { spheroid mixed } \\
\text { oxide }\end{array}$ & $-/ 38$ & $\begin{array}{l}100-200 \\
\mathrm{~nm}\end{array}$ & $34,38)$ \\
\hline $\begin{array}{l}\text { Variolink } \\
\text { Esthetic } \\
\text { DC }\end{array}$ & $\begin{array}{l}\text { Ivoclar } \\
\text { Vivadent }\end{array}$ & $\begin{array}{l}\text { UDMA, methacrylate } \\
\text { monomers, inorganic fillers, } \\
\text { initiators and stabilizers, } \\
\text { pigments }\end{array}$ & $\begin{array}{l}\text { spheroid mixed } \\
\text { oxide }\end{array}$ & $67 / 38$ & $\begin{array}{l}100-200 \\
\mathrm{~nm}\end{array}$ & $\begin{array}{l}34,38, \\
39)\end{array}$ \\
\hline $\begin{array}{l}\text { Multilink } \\
\text { Automix }\end{array}$ & $\begin{array}{l}\text { Ivoclar } \\
\text { Vivadent }\end{array}$ & $\begin{array}{l}\text { Dimethacrylate, HEMA, } \\
\text { barium glass filler, silica } \\
\text { filler, ytterbium trifluoride, } \\
\text { initiators, stabilizers, } \\
\text { pigments }\end{array}$ & $\begin{array}{l}\text { Barium glass filler, } \\
\text { silica filler, }\end{array}$ & $68.5 / 42.5$ & $\begin{array}{l}0.25-3.0 \\
\mu \mathrm{m}\end{array}$ & $40,41)$ \\
\hline $\begin{array}{l}\text { Panavia } \\
\text { V5 Paste }\end{array}$ & $\begin{array}{l}\text { Kuraray, } \\
\text { Tokyo, Japan }\end{array}$ & $\begin{array}{l}\text { Bis-GMA, TEGDMA, } \\
\text { hydrophobic aromatic } \\
\text { dimethacrylate, hydrophilic } \\
\text { aliphatic dimethac- } \\
\text { rylate, barium glass filler, } \\
\text { fluoroaluminosilicate glass, } \\
\text { silica filler, initiators, } \\
\text { stabilizers, pigments }\end{array}$ & $\begin{array}{l}\text { Barium glass filler, } \\
\text { fluoroaluminosilicate } \\
\text { glass, silica filler }\end{array}$ & $61 / 38$ & $\begin{array}{l}0.01-12 \\
\mu \mathrm{m}\end{array}$ & 40) \\
\hline $\begin{array}{l}\text { Tetric } \\
\text { EvoFlow }\end{array}$ & $\begin{array}{l}\text { Ivoclar } \\
\text { Vivadent }\end{array}$ & $\begin{array}{l}\text { Bis-GMA, UDMA, } \\
\text { ytterbiumtrifluorid, 1,10- } \\
\text { decandioldimethacrylat, } \\
\text { diphenyl-trimethylbenzoyl- } \\
\text { phosphinoxide }\end{array}$ & Barium glass filler & $68.1 / 43.8$ & $\begin{array}{l}0.04-3.0 \\
\mu \mathrm{m}\end{array}$ & 42) \\
\hline $\begin{array}{l}\text { Grey } \\
\text { Resin }\end{array}$ & $\begin{array}{l}\text { Formlabs, } \\
\text { Somerville, } \\
\text { MA, USA }\end{array}$ & $\begin{array}{l}\text { Methacrylated oligomer, } \\
\text { Methacrylated monomer, } \\
\text { Diphenyl (2,4,6- } \\
\text { trimethylbenzoyl) } \\
\text { phosphine oxid }\end{array}$ & - & - & - & 43) \\
\hline
\end{tabular}


the CAM software Preform (Formlabs) and printed on Form2 (Formlabs) with a grey acrylate resin (Formlabs). Postprocessing was carried out including the cleaning of the wheels for $3 \mathrm{~min}$ in isopropanol (96\%) activated with ultrasound and air-drying. Post-curing was performed by means of FormCure (Formlabs) for $30 \mathrm{~min}$ at $60^{\circ} \mathrm{C}$.

\section{Sample preparation and wear determination}

In general, ACTA sample wheels are divided into 20 test chambers, which are placed over the wheel circumference (14 mm length $\times 10 \mathrm{~mm}$ width $\times 4 \mathrm{~mm}$ thickness, Fig. 1 ). All chambers are numbered consecutively to avoid mixing-up the materials. All sample wheels were pre-treated with aluminum dioxide (50 $\mu \mathrm{m}, 1$ bar) to ensure a chemical frictional bond between the various materials. The luting materials ( $n=8$ for each material) were sequentially filled into the 20 compartments of each ACTA sample wheel (Table 1). In contrast to the polymer wheels, the steel wheels must be filled two millimeters higher, as the steel boundaries between the chambers would otherwise influence the abrasion. To enable the higher filling, two higher brass discs are fixed above and below the steel wheel. Since the composite of the 3D-printed wheels does not hinder the abrasion, a direct filling of the wheels was possible. Due to the photosensitivity of all materials, the chambers were light-cured separately for $40 \mathrm{~s}$ with a 1,000 $\mathrm{mW} / \mathrm{cm}^{2}$ curing lamp (SmartLite Focus, Dentsply Sirona, York, PA, USA). The remaining chambers were covered with a light-shield composite ring to ensure a unitary exposure time. Furthermore, the ambient light was reduced and the filled sample wheels were stored in a ringer's solution for $24 \mathrm{~h}$ at $37^{\circ} \mathrm{C}$.

Before the actual wear tests, the sample wheels were ground to compensate application inaccuracies

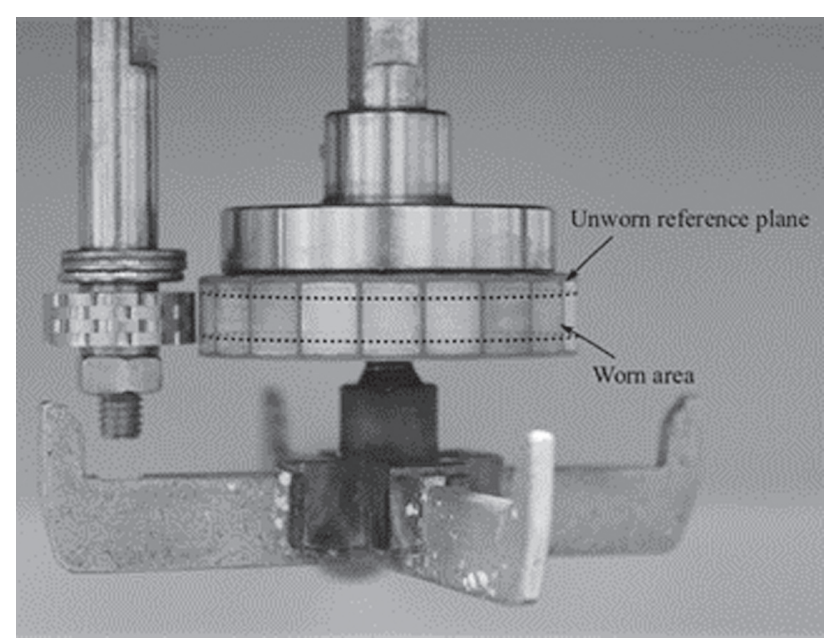

Fig. 1 Experimental set up of the ACTA-machine.

With a spring force of $15 \mathrm{~N}$ the antagonist wheel (left) was pressed against the sample wheel (right). The antagonist wheel is $4 \mathrm{~mm}$ smaller, creating a trace of abrasion and a reference plane. A stirrer attached to the axle below the sample wheel prevents the millet powder from settling. and to ensure a concentric running. Grinding wheels of different grain sizes are used to smoothen the surfaces. After storage and grinding, the sample wheel (right side) and the antagonist wheel (left side) were placed in the ACTA-machine. The antagonist wheel is made of stainless steel, has a honeycomb structure and is 2 $\mathrm{mm}$ smaller than the sample wheels to ensure unworn reference planes (Fig. 1). One-hundred-fifty grams of millet using the same batch during the entire experiment and $220 \mathrm{~mL}$ water were added and placed in a plastic case in which the two wheels run against each other. The abrasive mixture was changed every 50,000 cycles. To simulate the sliding movements and physiological forces appearing during chewing, the antagonist wheel rotated $15 \%$ slower than the sample wheel and was pressed against the sample wheel with a spring force of $15 \mathrm{~N}$.

After every 50,000 cycles, the wear was analyzed with a 3D Laser scanner (LaserScan3D, Willytec, Munich, Germany). The data obtained by the Laser scanning was processed with the Match 3D software (Gloger, Weinheim, Germany), which calculated the relative mean of the vertical height loss by comparing the worn area with the two unworn reference planes flanking the abraded area (Fig. 1). A total of 200,000 cycles per wheel was performed. The specimen wheels were kept wet between all steps.

\section{Field-emission-scanning-electron-microscope (FE-SEM) images}

After 200,000 cycles the worn surface of a selection of samples were morphologically analyzed with a FESEM (SEM Supra 55 VP, Carl Zeiss NTS, Oberkochen, Germany). In addition to the manufacturer's specifications the cured samples were burnt for $1 \mathrm{~min}$ in a flame in order to investigate filler differences of the materials. Further FE-SEM images were taken from the burnt samples.

\section{Finite element analysis}

The influence of the materials of the sample wheels on the wear behavior of the luting materials was investigated with a finite element analysis. In finite element analysis, a large structure is divided into several small simple-shaped elements. Thus, the individual deformation (stress) can be calculated more easily than for the whole undivided large structure ${ }^{24)}$. The structure of the ACTA sample wheels was reproduced using a CAD software (Solidworks Premium 2019 SP1.0, Dassault Systèmes). To perform the simulation, the material properties must be defined by the elastic modulus and the poisson's ratio. For the steel wheel an elastic modulus of $210 \mathrm{GPa}$ and a poisson's ratio of 0.28 , for the polymer wheel an elastic modulus of $2 \mathrm{GPa}$ and a poisson's ratio of 0.28 and for the luting agents an elastic modulus of $10 \mathrm{GPa}$ and a poisson's ratio of 0.28 was chosen. The simulation was carried out with the corresponding program Solidworks Simulation (Dassault Systèmes) assuming a vertical force of about $15 \mathrm{~N}$. 
Data were statically analyzed with R CRAN (R Core Team, Version 3.4.1) using ANOVA, post-hoc-Tukey-test and $t$-test. A $p$-value $<0.05$ was considered significant.

\section{RESULTS}

The main results are summarized in Table 2 and graphically plotted in Fig. 2. Using the polymer wheels, the wear values after 200,000 cycles ranged from $23.3 \pm 9.2$ $\mu \mathrm{m}$ (Tetric EvoFlow) to $27.8 \pm 6.2 \mu \mathrm{m}$ (Variolink Esthetic DC). Between 50,000 and 200,000 cycles, all materials showed a tripling of the wear values. While Multilink Automix showed the lowest, Variolink Esthetic DC exhibited the highest wear behavior after 200,000 cycles. The wear rate of all materials investigated were in a similar order of magnitude. With the polymer wheels, the luting agents investigated, exhibited no significant differences after each cycle (Table 2-homogenous subgroups $(\alpha=0.05))$.

Using the steel wheels, the same materials showed significant higher wear values as compared to the polymer wheels $(p<0.01, t$-test). After 200,000 cycles the

Table 2 Mean wear loss in $\mu \mathrm{m}$, SD, homogenous subgroups $(\alpha=0.05)$ and wear rate (nm/cycle) of all luting agents using polymer and steel sample wheels after 50,000, 100,000, 150,000, 200,000 cycles

\begin{tabular}{|c|c|c|c|c|c|c|c|}
\hline Material & & & $\begin{array}{c}\text { Variolink } \\
\text { Esthetic LC }\end{array}$ & $\begin{array}{c}\text { Variolink } \\
\text { Esthetic DC }\end{array}$ & $\begin{array}{l}\text { Multilink } \\
\text { Automix }\end{array}$ & $\begin{array}{l}\text { Panavia } \\
\text { V5 Paste }\end{array}$ & $\begin{array}{c}\text { Tetric } \\
\text { EvoFlow }\end{array}$ \\
\hline \multirow{6}{*}{$\begin{array}{l}\text { polymer } \\
\text { wheel }\end{array}$} & \multirow{5}{*}{ Wear } & \multicolumn{6}{|c|}{ No. of cycles } \\
\hline & & 50,000 & 10.5 [2.8] (a) & $10.3[2.8]$ (a) & 6.8 [2.5] (a) & 6.4 [2.6] (a) & 6.7 [1.8] (a) \\
\hline & & 100,000 & 13.1 [4.2] (a) & 15.7 [4.9] (a) & $11.3[2.6](\mathrm{a})$ & 11.8 [4.7] (a) & 10.7 [4.1] (a) \\
\hline & & 150,000 & $19.1[5.2]$ (a) & 21.9 [5.9] (a) & $16.4[5.3](a)$ & $17.3[6.6]$ (a) & 16.9 [5.4] (a) \\
\hline & & 200,000 & 27.2 [8.4] (a) & 27.8 [6.2] (a) & 23.2 [6.7] (a) & 24.8 [9.7] (a) & 23.3 [9.2] (a) \\
\hline & \multicolumn{2}{|c|}{ Wear rate } & 0.11 & 0.12 & 0.11 & 0.12 & 0.11 \\
\hline \multirow{6}{*}{$\begin{array}{l}\text { Steel } \\
\text { wheel }\end{array}$} & \multicolumn{3}{|c|}{ No. of cycles } & & & & \\
\hline & \multirow{4}{*}{ Wear } & 50,000 & $12.6[3.4](\mathrm{a})$ & $12.1[2.6](\mathrm{a})$ & 12.2 [4.2] (a) & 15.1 [3.9] (a) & 13.6 [5.6] (a) \\
\hline & & 100,000 & $30[20]$ (a) & 21.7 [7.7] (a) & 26.9 [9.8] (a) & 31 [10] (a) & 32 [14] (a) \\
\hline & & 150,000 & 53 [32] (a) & 32 [14] (a) & 43 [18] (a) & 46 [18] (a) & 58 [23] (a) \\
\hline & & 200,000 & 79 [37] (a) & 50 [16] (b) & 62 [20] (a) & 62 [22] (a) & 88 [24] (a) \\
\hline & \multicolumn{2}{|c|}{ Wear rate } & 0.00044 & 0.00025 & 0.00033 & 0.00031 & 0.0005 \\
\hline
\end{tabular}

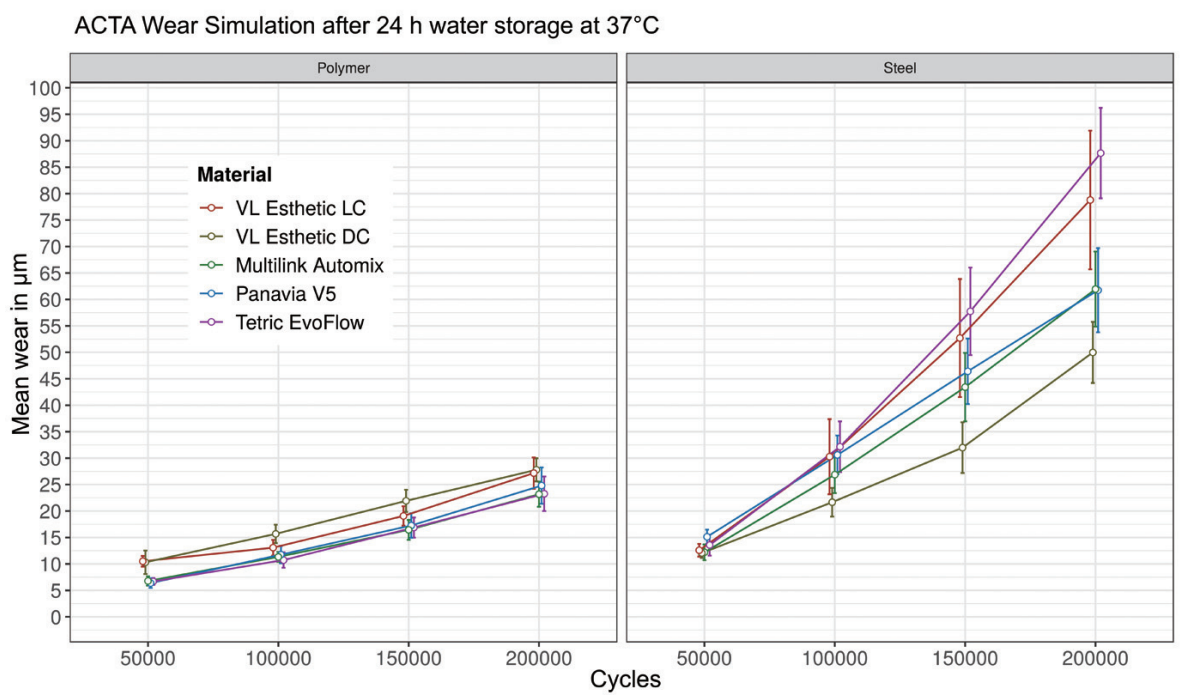

Fig. 2 Mean wear $(\mu \mathrm{m})$ with standard deviation as a function of cycles for all material investigated.

Using the polymer wheels, the materials investigated, exhibited no significant differences after each cycle. With the steel wheel Variolink Esthetic DC showed significant lower wear values compared to the reference material Tetric EvoFlow after 200,000 cycles $(p=0.037)$. 
values ranged from $50 \pm 16 \mu \mathrm{m}$ (Variolink Esthetic DC) to $88 \pm 24 \mu \mathrm{m}$ (Tetric EvoFlow).

Depending on the number of cycles an increase in wear values was observed. With 50,000-150,000 cycles wear showed no significant difference between the luting materials. After 200,000 cycles, wear values significantly increased to four (Variolink Esthetic DC) and even seven (Tetric EvoFlow) fold compared to the initial values. Wear rates were the lowest for Variolink Esthetic DC and highest for Tetric EvoFlow ( $p=0.037$, ANOVA).

Examples of the FE-SEM micrographs of all luting agents investigated are shown in Fig. 3. All images of the luting materials tested with the polymer wheel were blurred due to a smear layer and could hardly be used for surface analysis. In contrast, images of samples treated
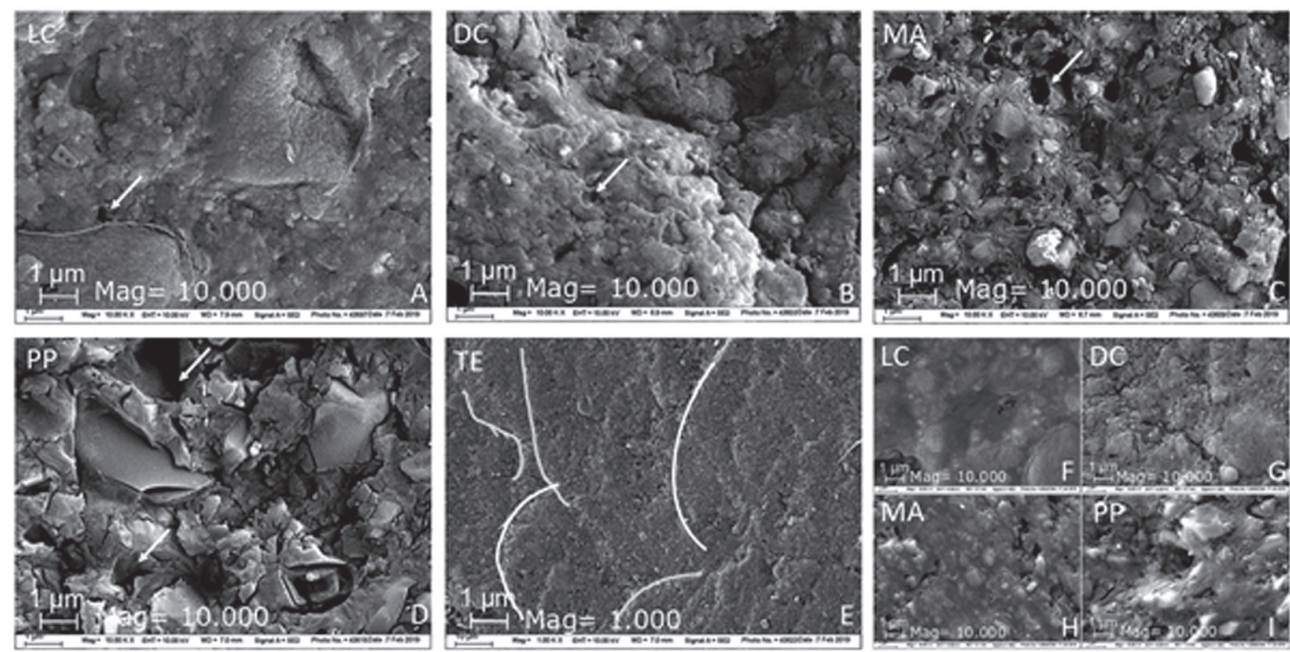

Fig. 3 FE-SEM micrographs of the worn surface after 200.000 cycles with a magnification of 1,000 and 10,000 .

White arrows shows defects caused by detached fillers; White lines (E) display the deformation of the materials in direction of the abrasion; A-E=Worn surface of the luting material testes with the steel wheel; F-I=Smeared surface of the luting material tested with the polymer wheel; LC=Variolink Esthetic LC; DC=Variolink Esthetic DC; MA=Multilink Automix; PP=Panavia V5 Paste; TE=Tetric EvoFlow

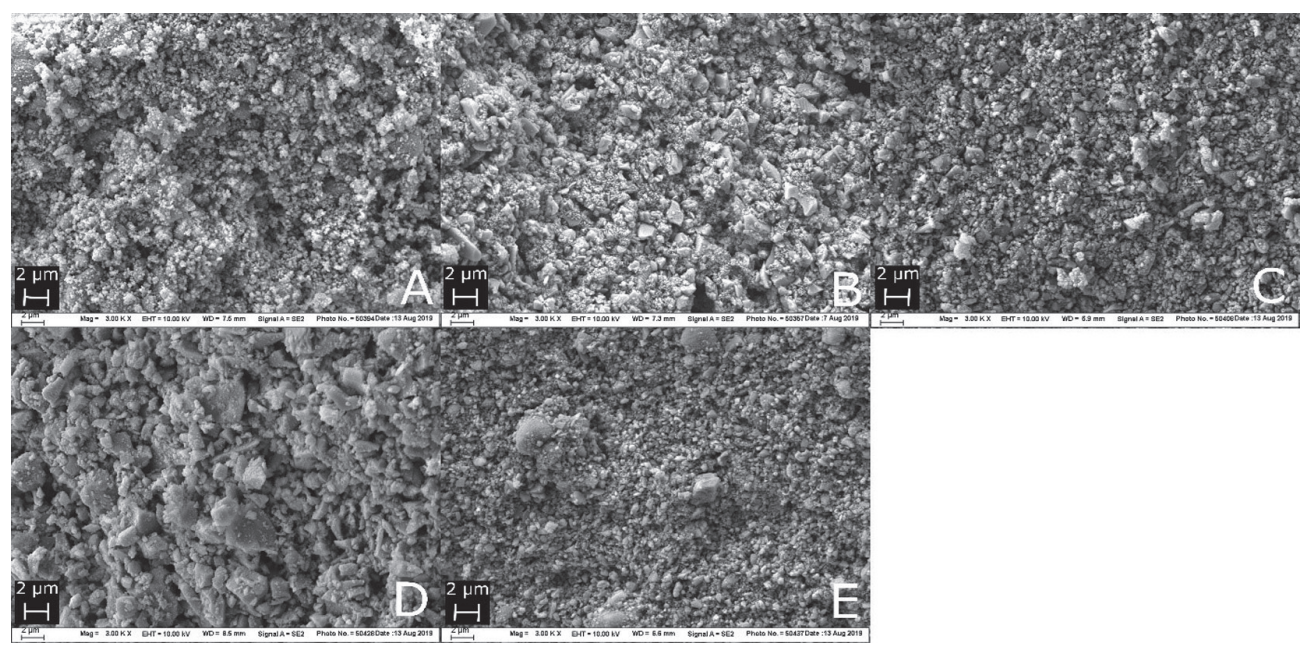

Fig. 4 FE-SEM micrographs of the burned samples of the materials investigated with a magnification of 10,000 .

A=Variolink Esthetic LC; B=Variolink Esthetic DC; C=Multilink Automix; D=Panavia V5 Paste; E=Tetric EvoFlow; Variolink LC and Variolink DC show comparable filler sizes $(0.1-3.0 \mu \mathrm{m})$. Mainly small fillers can be observed with isolated larger fillers up to $3 \mu \mathrm{m}$. Multilink Automix and Tetric EvoFlow exhibit a wide distribution of filler sizes of approx. 0.2-3.0 $\mu \mathrm{m}$, while Panavia V5 shows the largest filling sizes up to $5.0 \mu \mathrm{m}$. 

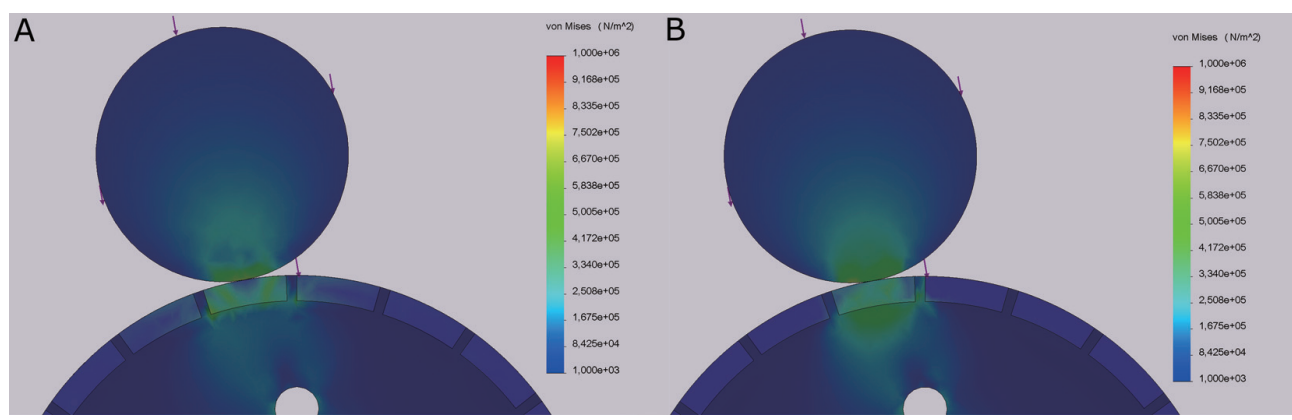

Fig. 5 Finite element analysis.

Distribution of the von Mises stress of the two sample wheels; Polymer wheel (A) shows lower stress within the samples compared to the steel wheel (B).

by the steel wheel showed a clear appearance. A wide variety of material-dependent filler sizes were observed. Variolink Esthetic LC and Variolink Esthetic DC (Figs. $3 \mathrm{~A}, \mathrm{~B})$ showed the smallest sizes of about $0.1 \mu \mathrm{m}$. The largest fillers were observed with Panavia V5 Paste (up to $8.0 \mu \mathrm{m}$ ). Notably, Multilink Automix (Fig. 3C) showed a wide range of irregular shaped fillers with a size from 0.5-1.5 $\mu \mathrm{m}$. The reference material Tetric EvoFlow (Fig. $3 \mathrm{E})$ exhibited a wave-like patterns resembling the shift of the composite in the direction of the abrasion. In general, the matrix was partially eroded, and voids were observed due to the filler exfoliation. Scratches on the surface or abrasion particles of the millet suspension in form of photolytes were not observed.

Figure 4 shows the FE-SEM images of the burned samples. No differences in filling size $(0.1-3.0 \mu \mathrm{m})$ and filler distribution between Variolink LC and Variolink DC were detected (Figs. 4A, B). The two materials mainly showed small fillers with isolated larger fillers up to $3.0 \mu \mathrm{m}$. Both Multilink Automix and Tetric EvoFlow exhibited a wider distribution of filler sizes of approx. 0.2-3.0 $\mu \mathrm{m}$ (Figs. 4C, D). The largest filler sizes up to 5.0 $\mu \mathrm{m}$ were observed with Panavia V5.

Figure 5 displays an example of the stress distribution of the two sample wheels of the experimental setup simulated with a finite element analysis. Force per area $\left(\mathrm{N} / \mathrm{m}^{2}\right)$ was in a similar range, but the steel wheel showed a wider and deeper force transmission, which was concentrated in the center of the samples. The polymer wheel exhibited reduced stress, which was located at the edge of the chambers.

\section{DISCUSSION}

In analogy to the first publications by De Gee et al., the sample wheels of the ACTA wear simulation machine have been made of stainless steel for more than three decades $^{20)}$. When the ACTA machine was developed in 1986, it was not possible to establish a chemical bond between the composites to be tested and the ACTA steel wheel $^{20)}$. In order to achieve a mechanical retention, steel boundaries were insert in the ACTA sample wheels. However, these steel boundaries themselves have an influence on the wear behavior, as the steel boundaries show a much higher Young's modulus compared to the Young's modulus of the tested composites ${ }^{20,25,26)}$. For that reason, that the steel boundaries have no longer any influence on the stresses at the specimen surface, the composite thickness has been increased by $2 \mathrm{~mm}$ for more than two decades in our research facility. However, therefore twice as much composite is required for each test, making this solution more cost intensive and complicated.

The possibility to print ACTA wheels is a promising way to avoid these disadvantages. Printing materials are cheap and allow a chemical bond with composites. Moreover, an individual design of the 3D printing ACTA wheel compartments can be created. As the 3D printed wheels can be worn off with the composite, an easy filling of the sample wheels is possible. In addition, it was originally assumed that the $3 \mathrm{D}$ printed ACTA wheels would not have any influence on wear behavior as the Young's modulus of the material is similar or lower than the composites investigated. Furthermore, most of the $3 \mathrm{D}$ printed materials do not contain fillers that could influence the wear behavior ${ }^{26-28)}$.

In the first step of our experiments, we replaced conventional steel ACTA wheels with 3D printed polymer versions. Unexpectedly, the material of the sample wheels had a much higher influence on wear behavior, as the modified set up exhibited much lower wear values despite comparable resins in previous studies ${ }^{19,23)}$.

The FE-SEM images of the polymer wheels could hardly be analyzed due to a smear layer. It remains unclear which factors caused the smear layer. One hypothesis is the formation of a wax film created by the millet suspension. Pallav et al. reported a disturbing wax layer on the surface of the sample wheels caused by fat released by the millet seeds during the experiment (fat content $3-4 \mathrm{wt} \%)^{29)}$. Therefore, they recommended to renew the millet suspension every 50,000 cycles to reduce the fat film as we have done in our experiments. Moreover, millet contains plant stones, so-called phytoliths, which would have been present in the FESEM images if millet components were imprinted in the materials investigated $^{30)}$. Since we were not able to detect any phytoliths in the FE-SEM images and a smear layer was only observed on images of materials tested with 
polymer wheels, the theory of a wax formation due to the millet suspension appears unlikely.

Alternatively, the smear layer may be caused by a so-called tribofilm, which develops as a compacted surface on top of composite materials as worn particles are clenched into the matrix. A tribofilm is harder than composite materials and is characterized by less friction, stress and wear ${ }^{31)}$. The 3D printed polymer wheels used in our initial experiments show a low elasticity modulus in contrast to the steel wheels (tensile modulus 2.8 GPa, flexural modulus 2.2 GPa, Fig. 5). This implies a lower and more superficial force transmission by the antagonist wheel due to a deformation of the polymer wheel, resulting in less material abrasion. By contrast, the high elasticity modulus of the steel wheel resulted in a higher and deeper force transmission and an increased material loss compared to the polymer wheels (Fig. 5). The reduced and superficial power transmission due to the deformation of the polymer wheel leads to an exfoliation of smaller particles in contrast to the steel wheel (Fig. 6A). These small abrasion particles can be deposited in the spaces between the fillers (Fig. 6Aright) and create a smear layer due to the compression of the small particles into the matrix. In contrast, using the steel wheel, the increased force caused larger particles to break out (Fig. 6A-left). The larger particles cannot be deposited between the fillers and are worn off, resulting in increased abrasion and a lack of a tribofilm development. Earlier studies postulated, that a tribofilm can be avoided by an abrasive medium like the millet suspension ${ }^{32}$. Since none of these studies used an experimental setup with 3D printed polymer wheels before, a formation of a tribofilm caused by the low elasticity modulus of the polymer wheel is the most probable explanation.

In general, all investigated luting agents tested with the steel wheel exhibited significant higher wear values than those tested with the polymer wheel ( $p<0.001, t$-test), due to a lower and more superficial force transmission by the antagonist wheel due to a deformation of the polymer wheel, resulting in less material abrasion, as described in detail above (Fig. 6A). Unlike with steel wheels no significant differences between the materials were observed using the polymer wheels. Therefore, we concluded, that this type of 3D-printing material used in this study should not be used to replace the classic steel wheels as they do not correctly reflect the wear behavior of the luting materials investigated.

After 200,000 cycles using the steel wheel, Variolink Esthetic DC showed significantly lower wear values $(p=0.037$, ANOVA) as compared to the reference material Tetric EvoFlow. It has been shown that filler-load, -size and-distribution have an influence on the physical and mechanical properties of luting composites ${ }^{33-35)}$. Jørgensen and Baynes postulated that small fillers and high filler load offer better protection against abrasion. If small fillers are homogeneously distributed and closely packed, the softer matrix is protected from abrasion and wear is reduced (Fig. 6B) ${ }^{36,37)}$. Variolink DC, which achieved the best wear result, exhibits the

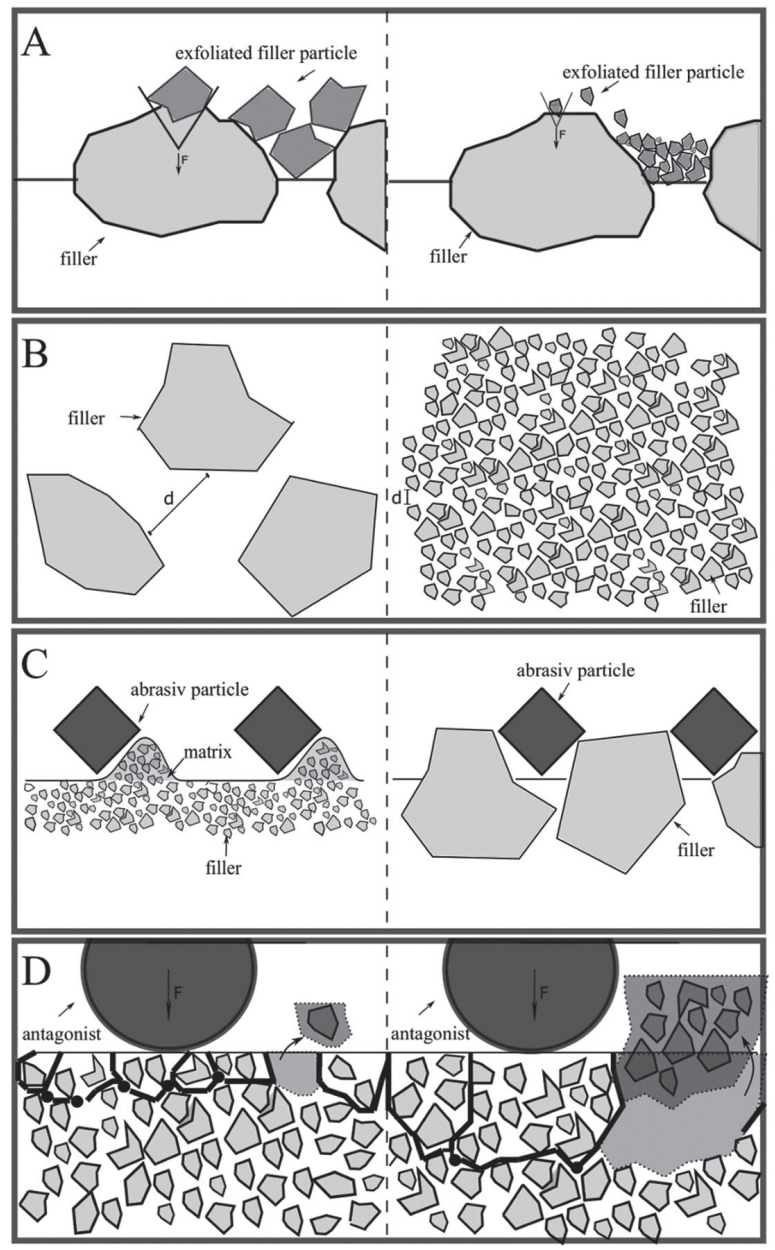

Fig. 6 Schematic illustration of the relationship between wear and material properties.

A: The low E-modulus of the polymer wheel resulted in a lower force transmission causing an abrasion of smaller particles which are deposited in the spaces between the fillers (left). The high E-modulus of the steel wheel resulted in a higher force transmission. Larger particles are broken out by which cannot fit between the filler gaps and are washed out (right). B: Principle of micro-protection according to Jørgensen. The narrow distance between the small fillers (right) causes a better protection of the softer matrix than the wide distance through the large fillers (left). C: Relationship between the size of the abrasive particles and the filler size (Yuasa 1990). Fillers smaller than the abrasive particles are removed along with the matrix. Wear protection only occurs when the filler is of a comparable size to the abrasive particles. D: Schematic representation of the formation of sealing particles according to Shuh's delamination theory (1986). Depending on the elastic modulus of the material, the microcavities are located at different depths. Left: Material with high elastic modulus (e.g. Variolink DC); The cracks are close to the surface and small particles are dissolved. Right: Material with low elastic modulus (e.g. Variolink LC). The cracks are in deeper planes, causing larger particles to be dissolved. 
lowest filling load (38 vol\%) and the smallest filler size (100-200 nm). In contrast, Tetric EvoFlow showing significantly higher wear values compared to Variolink DC, exhibits the highest filler load (43.8 vol\%) and one of the largest fillers $(0.04-3 \mu \mathrm{m})$ (Table 1). These findings are in line with the theory of Jørgensen and Baynes postulating that the smaller particles from Variolink LC may protect the matrix against abrasion. For all materials investigated in our experiments, the size of filler particles (Variolink DC=Variolink LC $<$ Multilink Automix $<$ Tetric EvoFlow $<$ Panavia V5 Paste) basically correlated negatively to wear values measured (Variolink DC $<$ Panavia V5 Paste $<$ Multilink Automix $<$ Variolink $\mathrm{LC}<$ Tetric EvoFlow). The smaller the particles the higher the wear resistance. In contrast to Baynes' theory that a higher filler load results in lower wear, the rank order of the filler load of all luting materials in vol\% (Tetric EvoFlow $>$ Multilink Automix $>$ Panavia V5 Paste $=$ Variolink LC=Variolink DC) did not correlate with the measured wear values (Variolink $\mathrm{DC}<$ Panavia V5 Paste $<$ Multilink Automix $<$ Variolink LC $<$ Tetric EvoFlow) but showed rather a contrary trend. Thus, it appears that the filler load has a neglectable influence on the wear of the luting materials.

Although filler sizes and wear values, in general, showed a positive correlation, this was not consistent for all materials. Variolink LC exhibited poor wear values despite a small filler size (100-200 nm), while Panavia V5 showed one of the lowest wear results despite a large filler size $(0.01-12 \mu \mathrm{m})$. Yuasa emphasized that the ratio of filler size to abrasive particles is another important factor influencing the wear ${ }^{38)}$. If the abrasive particles are larger than the containing fillers, the wear is determined by the softer matrix (Fig. 6C-left). If the abrasive particles are smaller than the filler, matrix protection effect is achieved, and wear is determined by the filler (Fig. 6C-right). As the phytoliths (abrasive particles of millet) exhibit a size of approx. 9-12 $\mu \mathrm{m}$, almost all investigated materials showed smaller fillers (Table 1). The only exception was Panavia V5 paste which showed a filler size of up to $12 \mu \mathrm{m}$ (Fig. 3D) and thus, exhibited one of the best abrasion values (Fig. 2).

Unexpectedly, Variolink LC showed the worst result besides the reference material Tetric EvoFlow. Even though the manufacturers describe the same production of Variolink LC and Variolink DC with the same filler size and filler content different wear results were observed. In order to investigate possible differences in filler-size and -distribution, despite the manufacturer's specifications, specimens of both materials were additionally burnt out. Larger fillers (up to $3 \mu \mathrm{m}$ ) than specified by the manufacturer $(100-200 \mathrm{~nm})$ were observed in the FE-SEM surface images of the burnout, however, no differences in filler size or distribution between Variolink LC and Variolink DC were detected (Fig. 5).

The manufacturer described a lower elastic modulus of Variolink LC (4160 $\pm 169 \mathrm{MPa})$ in contrast to Variolink DC $(6570 \pm 290 \mathrm{MPa})^{39}$. Earlier studies were able to prove that a the filler degree, hardness, and elastic modulus exhibit an influence on the wear behavior of dental materials ${ }^{40,41)}$. According to Suh, the underlying micro mechanism is explained by the elastic and plastic deformation of the material under cyclic load ${ }^{42}$. Stress increases in deformed materials, resulting in gaps or cracks as soon as the applied load exceeds the material strength. The cracks enlarge until wear particles are broken out. A high elastic modulus exhibit near-surface stress maxima with small wear particles dissolving, resulting in small surface defects (Fig. 6D-left). A low elastic modulus shows stress at deeper planes. Larger particles are dissolved, resulting in increased wear (Fig. 6D-right). Thus, the low elasticity modulus of Variolink LC causes bigger particles to break out resulting in higher wear values compared to Variolink DC, despite identical production. The manufacturer's data do not allow to figure out which factor may be responsible for the different moduli of elasticity.

\section{CONCLUSION}

The dual- or auto-polymerizing materials (Variolink Esthetic DC, Multilink Automix, Panavia V5) have a small, not significant advantage compared to the light curing materials (Variolink Esthetic LC) regarding the wear resistance. This type of $3 \mathrm{D}$ printing material used in this study should not be used to replace the classic steel wheels as the material of the sample wheels has more influence than expected. To extend the classic test setup of the ACTA machine, further tests should be conducted with other 3D printing materials preferably with a higher elasticity modulus.

\section{REFERENCES}

1) Brockman H, Serres F de, Barnett W. Analysis of ad-3 mutants induced by nitrous acid in a heterokaryon of Neurospora crassa. Mutat Res 1993; 7: 307-314.

2) Mitchell C, Pintado M, Douglas W. Nondestructive, in vitro quantification of crown margins. J Prosthet Dent 2001; 85: 575-584.

3) Backer H, van Maele G, Moor N, van den Berghe L, Boever J. A 20-year retrospective survival study of fixed partial dentures. Int J Prosthodont 2006; 19: 143-153.

4) Goldman M, Laosonthorn P, White R. Microleakage - full crowns and the dental pulp. J Endod 1992; 18: 473-475.

5) Goldin E, Boyd N, Goldstein G, Hittelman E, Thompson V. Marginal fit of leucite-glass pressable ceramic restorations and ceramic-pressed-to-metal restorations. J Prosthet Dent 2005; 93: 143-147.

6) Pettenò D, Schierano G, Bassi F, Bresciano M, Carossa S. Comparison of marginal fit of 3 different metal-ceramic systems: an in vitro study. Int J Prosthodont 2000; 13: 405408.

7) White S, Sorensen J, Kang S, Caputo A. Microleakage of new crown and fixed partial denture luting agents. J Prosthet Dent 1992; 67: 156-161.

8) Krämer N, Frankenberger R. Leucite-reinforced glass ceramic inlays after six years: wear of luting composites. Oper Dent 2000; 25: 466-472.

9) van Dijken JWV, Hörstedt P. Marginal breakdown of fired ceramic inlays cemented with glass polyalkenoate (ionomer) cement or resin composite. J Dent 1994; 22: 265-272.

10) McLean J, Fraunhofer J von. The estimation of cement film 
thickness by an in vivo technique. Br Dent J. 1971; 131: 107111.

11) Malament K, Socransky S. Survival of Dicor glass-ceramic dental restorations over 16 years. Part III: effect of luting agent and tooth or tooth-substitute core structure. J Prosthet Dent 2001; 86: 511-519.

12) Kessler A, Reymus M, Hickel R, Kunzelmann K-H. Threebody wear of 3D printed temporary materials. Dent Mater 2019; 35: 1805-1812.

13) Harsha AP, Tewari US. Two-body and three-body abrasive wear behaviour of polyaryletherketone composites. Polymer Testing 2003; 22: 403-418.

14) Lambrechts $P$, Vanherle G, Vuylsteke $M$, Davidson C. Quantitative evaluation of the wear resistance of posterior dental restorations: a new three-dimensional measuring technique. J Dent 1984; 12: 252-267.

15) McCabe J, Molyvda S, Rolland S, Rusby S, Carrick T. Twoand three-body wear of dental restorative materials. Int Dent J 2002; 52: 406-416.

16) Ehrnford L, Dérand T, Larsson L, Svensson A. An abrasion test for composite resins. J Dent Res 1980; 59: 716-720.

17) Bailey W, Rice S. Comparative sliding-wear behavior of a dental amalgam and a composite restorative as a function of contact stress. J Dent Res 1981; 60: 731-732.

18) Fan PL, Powers JM, Craig RG. In vitro wear of microfilled and visible light-cured composites. J Dent Res 1979; 58: 21162119.

19) Gee A de, Pallav P. Occlusal wear simulation with the ACTA wear machine. J Dent 1994; 22: 21-27.

20) Gee A de, Pallav P, Davidson C. Effect of abrasion medium on wear of stress-bearing composites and amalgam in vitro. J Dent Res 1986; 65: 654-658.

21) Fang H. Optimization and standardization of the Erlangen system for simulation of contact wear of direct restorations. Doctoral dissertation (in German), Ludwigs-Maximillians Universitiy Munich, Germany (1992).

22) Sobota M. In-vitro wear of experimental composites with variation of the filler content, the filler size and the antagonist size and shape. Doctoral dissertation (in German), LudwigsMaximillians Universitiy Munich, Germany (2001).

23) Say E, Civelek A, Nobecourt A, Ersoy M, Guleryuz C. Wear and microhardness of different resin composite materials. Oper Dent 2003; 28: 628-634.

24) Magne P. Efficient 3D finite element analysis of dental restorative procedures using micro-CT data. Dent Mater 2007; 23: 539-548.

25) Grünwald A. Food wear and material fatigue of experimental filling materials. Doctoral dissertation (in German), LudwigsMaximillians Universitiy Munich, Germany (2001).

26) Reich S, Petschelt A, Wichmann M, Frankenberger R.
Mechanical properties and three-body wear of veneering composites and their matrices. J Biomed Mater Res A 2004; 69: $65-69$.

27) Kunzelmann KH. Glass-ionomer cements, cermet cements,"hybrid"-glass-ionomer and compomer-laboratory trials—wear resistance. Acad Dent Mater 1996; 9: 89-104.

28) Bauer CM, Kunzelmann KH, Hickel R. Simulated food wear of resin composites and ormocers. Dtsch Zahnarztl Z 1995; 50: 635-638 (in German, abstract in English).

29) Pallav P, Gee A de, Werner A, Davidson C. Influence of shearing action of food on contact stress and subsequent wear of stress-bearing composites. J Dent Res 1993; 72: 56-61.

30) Guegel I, Grupe G, Kunzelmann KH. Simulation of dental microwear. Am J Phys Anthropol 2001; 114: 124-138.

31) Komvopoulos K, Li H. The effect of tribofilm formation and humidity on the friction and wear properties of ceramic materials. J Tribol 1991; 114: 131-140.

32) Mair M. Food wear in a chewing simulator. Doctoral dissertation (in German), Ludwigs-Maximillians Universitiy Munich, Germany (2002).

33) Venhoven B, de Gee A, Werner A, Davidson C. Influence of filler parameters on the mechanical coherence of dental restorative resin composites. Biomaterials 1996; 17: 735-740.

34) Leinfelder K. Posterior composites. State-of-the-art clinical applications. Dent Clin North Am 1993; 37: 411-418.

35) Hill E, Lott J. A clinically focused discussion of luting materials. Aust Dent J 2011; 56: 67-76.

36) Bayne S, Taylor D, Heymann H. Protection hypothesis for composite wear. Dent Mater 1992; 8: 305-309.

37) Jørgensen KD, Hørsted P, Janum O, Krogh J, Schultz J. Abrasion of Class 1 restorative resins. Eur J Oral Sci 1997; 87: 140-145.

38) Yuasa S. Influences of composition on brush wear of composite resins. Influences of particle size and content of filler. Shika Zairyo Kikai 1990; 9: 659-678.

39) Ivoclar vivadent Variolink Esthetic - The esthetic luting composite. (2019. www.ivoclarvivadent.com/zoolu-website/ media/document/38411/Variolink+Esthetic+-+Ivoclar+ Vivadent+Report+No-+22. Accessed Tuesday, September 10, 2019.

40) Kunzelmann K. Wear analysis and quantification of restorative materials in vivo and in vitro. 1th ed. Aachen, Germany: Shaker Verlag; 1998.

41) Braem M, Finger $W$, van Doren $V$, Lambrechts $P$, Vanherle G. Mechanical properties and filler fraction of dental composites. Dent Mater 1989; 5: 346-348.

42) Suh N. Tribophysics. Englewood Cliffs, NJ: Prentice Hall; 1986.

43) Dukic W. Radiopacity of composite luting cements using a digital technique. J Prosthodont 2017; 28: 450-459. 